\title{
Non-small cell lung cancer invasion and metastasis promoted by MMP-26
}

\author{
YANG ZHANG $^{1 *}$, HANG ZHAO $^{2,5^{*}}$, YELING WANG ${ }^{3}$, YONG LIN $^{5}$, \\ YAN TAN $^{2}$, XUEXUN FANG $^{4}$ and LIANWEN ZHENG

\begin{abstract}
${ }^{1}$ School of Pharmaceutical Sciences; ${ }^{2}$ Central Laboratory of the First Hospital; ${ }^{3}$ Department of Vasculocardiology, the First Hospital of Jilin University; ${ }^{4}$ Key Laboratory for Molecular Enzymology and Engineering, Ministry of Education, Jilin University, Changchun 130021; ${ }^{5}$ The Central Hospital of Changchun City, Changchun 130051;

${ }^{6}$ Reproductive Center of the Second Hospital, Jilin University, Changchun 130041, Jilin, P.R. China
\end{abstract}

Received March 22, 2011; Accepted July 19, 2011

DOI: $10.3892 / \mathrm{mmr} .2011 .540$

\begin{abstract}
Matrix metalloproteinase 26 (MMP-26) is a novel member of the matrix metalloproteinase (MMP) family and is widely expressed in cancer cells of epithelial origin. MMP-26 has been shown to contribute to tumor development and to the restoration of tissue injury. In this study, in order to identify the functions of MMP-26 that contribute to the biological phenotype and behavior of human lung carcinoma A549 cells, we established an MMP-26 low-expressing tumor cell model using RNA interference (RNAi) transfection. These cells were used to investigate the role of MMP-26 in tumor progression. The MTT, colony forming, adherence and spreading, woundhealing and Transwell chamber invasion assays were performed to analyze the invasion ability of pshRNA-MMP26-transfected A549 cells. Semi-quantitative reverse transcription polymerase chain reaction, Western blotting and double immunofluorescent staining were employed to detect the relationship between MMP-26 and MMP-9. Results showed that the adhesive rate was down-regulated in pshRNA-MMP26-transfected cells, compared to the controls. Silencing of the MMP-26 gene significantly retarded the invasiveness of A549 cells in Transwell insert invasion assays. The cells proliferated in the three-dimensional (3D) culture system. A549 cells transfected with the pshRNA-MMP26-C plasmid mainly developed reticular structures in morphology, and formed few clones with clear and smooth edges as well as tight intercellular junctions. The mRNA and protein expression of MMP-9 in pshRNA-MMP26-transfected cells were significantly lower than those of the controls. Double immunofluorescence labeling
\end{abstract}

Correspondence to: Dr Lianwen Zheng, Reproductive Center of the Second Hospital, Jilin University, Changchun 130041, Jilin, P.R. China

E-mail: zyang@jlu.edu.cn; zhenglw@jlu.edu.cn

*Contributed equally

Key words: lung neoplasms, matrix metalloproteinases, invasion and focal laser scanning microscopy showed that MMP-26 was colocalized with MMP-9 in the controls. In conclusion, we successfully established an MMP-26 low-expressing cell model and confirmed that MMP-26 contributed to A549 cell invasion and migration in vitro. We also demonstrated that MMP-26 plays an important role in local invasion at least in part through coordination with MMP-9.

\section{Introduction}

Lung cancer is characterized by high morbidity and mortality. Non-small cell lung cancer (NSCLC) accounts for $80 \%$ of new cases of lung cancer. According to the latest statistics provided by the WHO international cancer research center in 2004, the total incidence of lung cancer was $20 \%$ in all cancer patients and the mortality rate was $23.8 \%$ of cancercaused deaths (1). Despite progress in surgery, radiotherapy and chemotherapy in recent years, the 3 -year survival rates of phase II and phase III NSCLC patients remains at only $35-50 \%$ and $28 \%$, respectively (2). The majority of patients succumb to local recurrence or distant metastasis. Therefore, understanding the process of invasion and metastasis of NSCLC at the molecular level, and finding reliable markers to predict recurrence and adverse prognosis constitute the focus of current investigations.

Matrix metalloproteinase 26 (MMP-26) is a new member of the MMP family and also a human-specific protease. It is capable of effectively degrading many extracellular matrix (ECM) components such as fibronectin, collagen IV, vitronectin, and fibrinogen, and it may also activate pro MMP-9. MMP-26 is expressed in epithelial tumors such as breast, endometrial and prostate cancer, as well as their corresponding cell lines (3-6). Additionally, MMP-26 may play a significant role in the processes of tumor invasion and metastasis.

At present, the MMP-26 expression in NSCLC and its significance are poorly understood. Therefore, the effect of MMP-26 on cell invasion and its pathway were studied by gene silencing and transfection in A549 cells (3), which provide a model of NSCLC with a high expression of MMP-26. The aim 
of this study was to investigate the role of MMP-26 in NSCLC invasion, as well as its action pathway.

\section{Materials and methods}

Construction of recombinant plasmid with stable expression. Three pairs of oligonucleotides targeting the coding regions (181-199, 560-578 and 952-970 bp) were designed and synthesized according to the MMP-26 mRNA sequences (NM_021801) in Genbank and the design principles of small interfering RNA (siRNA) (7). In addition, RNA interference (RNAi) sequences (84-102 bp, AF420592) for green fluorescent protein (GFP) that were unrelated to the sequences of MMP-26 were used as negative controls. The RNAi sequence $(1,696-1,714 \mathrm{bp}, \mathrm{BC} 013380)$ for the housekeeping gene $\beta$-actin was used as a positive control. The sequences were as follows: pshRNA-MMP26 A: 5'-GATCCCCACACAGCTCCTGCAACAATTTCA AGAGA ATTGTTGCAGGAGCTGTGTTTTTTA-3', 3'- GGGTGTGTCGAGGACGTTGT TAA AGT TC TCTTAACAACGTCCTCGACACAAAAAATTCGA-5'; pshRNA-MMP26-B: 5'-GATCCCCATCCTGGAGTTGTC CATTTTTCAAGAGAAAATGGACA ACTCCAGGAT TTTTTA-3', 3'-GGGTAGGACCTCAACAGGTAAAAAG T TC TCT TTTACCTGTTGAGGTCCTAAAAAT T CGA-5'; pshRNA-MMP26-C: 5'-GATCCCCAACGCTA CTGAGTCACAATT TCA A GAGA A T TGTGAC TCAGTAGCGTTTTTTTA-3', 3'-GGGTTCGATGACTC AGT TT TAA AG T T C T C T TA ACACTGAGTCA TCGCAAAAAATTCGA-5'; pshRNA-GFP: 5'-GATCCCC GAA ACCATGCAA A G TA A GGT T C A AGAGA C C T TACT T T GCATGG T T T C T T T T T A - 3 ', 3'-GGGCTTTGGTACGTTTCATTCCA AGTTCTCT GGAATGAAACGTACCAAAGA A A AATTCGA-5'; pshRNA- $\beta$-actin: 5'-GATCCCCCTTGAGATGTATGAAGG CT T TCA AGAGA A GCC T TCATACATCTCA AG TTTTTA-3', 3'-GGGGAACTCTACATACTTCCGAAAG TTCTCTTCGGAAGTATGTAGAGTTC AAAAATTCGA-5'.

pSUPERIOR.puro was digested with BglII and HindIII (Takara, Japan). After purification, a linear vector was formed. Under the action of T4 ligase, the vector was connected to the above oligonucleotide following annealing and a recombinant vector was formed. The recombinant vector was transformed and amplified and the plasmid was then extracted. Following confirmation by EcoRI and HindIII (Takara), positive clones were sequenced (Shanghai Biological Engineering Co., Ltd., China).

Cell culture and transfection. The human carcinoma cell line A549 was provided by the Biological Engineering Center of the School of Pharmaceutical Sciences, Jilin University, China. A549 cells were cultured in Dulbecco's modified Eagle's medium (DMEM) (Gibco, Carlsbad, CA, USA) containing $10 \%$ fetal bovine serum at $37^{\circ} \mathrm{C}$ and $5 \% \mathrm{CO}_{2}$. The recombinant vector was transfected into A549 cells by liposome-mediated transfection $24 \mathrm{~h}$ after seeding. At the same time, the empty vector and PBS-treated control groups were set. The specific operation was in accordance with the instructions in the lipofectamine ${ }^{\mathrm{TM}} 2000$ transfection kit (Invitrogen, Carlsbad, CA, USA). After 12, 24, 36, 48 and $72 \mathrm{~h}$ transfection, the cells were collected.
Semi-quantitative RT-PCR. The total RNA in different groups was extracted with the TRIzol reagent. The concentration and purity were determined by ultraviolet spectrophotometry. According to the one-step reverse transcription polymerase chain reaction (RT-PCR) kit (MBI Fermentas, Glen Burnie, MD, USA) instructions, the total RNA was used as a template, and reverse transcriptase synthesis of the cDNA was performed. The product underwent semi-quantitative PCR. $\beta$-actin was set as the internal reference. The primer was synthesized by Shanghai Shenggong Co., Ltd, China. The MMP-26 primer sequences were: upstream 5'-GGGACTTTGTTGAGGGCTAT-3', downstream 5'-CTGG CGAGATGGAGGTGT-3'; the length of the amplification product was $198 \mathrm{bp}$. The MMP-9 primers sequences were: upstream 5'-GTGCTGGGCGCTGCTTTGCT-3', downstream 5'-GTCGCCCTCAAAGGTTTGGAA-3'; the length of amplification product was $350 \mathrm{bp}$. The $\beta$-actin primers sequences were: upstream 5'-CACCCTGTGCTGCTCACCGAGCC-3', downstream 5'-CCACACAGATGACTTGCGCTCAGG-3'; the length of the amplification product was $690 \mathrm{bp}$. The PCR reaction conditions were $3 \mathrm{~min}$ of predenaturation at $94^{\circ} \mathrm{C}$, $45 \mathrm{sec}$ of denaturation at $94^{\circ} \mathrm{C}, 1 \mathrm{~min}$ of annealing at $53^{\circ} \mathrm{C}$, $1 \mathrm{~min}$ of extension at $72^{\circ} \mathrm{C}$, with a total of 35 cycles, followed by $5 \mathrm{~min}$ of extension at $72^{\circ} \mathrm{C}$. The product underwent $1.5 \%$ agarose gel electrophoresis, and the band was scanned and visualized with a gel imaging analysis system. The ratio of the target gene and $\beta$-actin was then calculated. Each experiment was repeated 3 times.

Western blot analysis. Treated cells in different groups were collected, the total protein was extracted from lysed cells, and protein determination was performed. A 10\% sodium dodecyl sulfate polyacrylamide gel electrophoresis (SDS-PAGE) was prepared. The loading sample for each well was $50 \mu \mathrm{g}$. Following electrophoresis, membrane transfer and blocking were performed. The membrane was then reacted with MMP-26 polyclonal antibody (1:500), or MMP-9 polyclonal antibody (Sigma-Aldrich, St. Louis, MO, USA) (1:400) and horseradish peroxidase-labeled secondary antibodies $(1: 2,500)$ for $1 \mathrm{~h}$. DAB coloring was finished within $30 \mathrm{~min}$. Each experiment was repeated 3 times.

Determination of growth curve. Cells in the logarithmic phase were collected and single cell suspensions were prepared. The cells were seeded in 96-well plates with $2.0 \times 10^{4}$ cells in each well. Then, $20 \mu 1$ of MTT solution (Sigma, St. Louis, MO, USA) $(5 \mathrm{mg} / \mathrm{ml})$ was added $24,48,72,96,120$ and $144 \mathrm{~h}$ after culture. After a further $4 \mathrm{~h}$ of culturing, the supernatant was discarded and $150 \mu \mathrm{l}$ of dimethyl sulfoxide was added for action termination. The optical density value at $490 \mathrm{~nm}$ was determined using a microplate reader and a cell growth curve was performed. Each experiment included 6 samples and the average value was used.

Colony forming assay. Cells in the logarithmic phase were collected and single cell suspensions were prepared. The cells were seeded in 6-well plates with 200 cells in each well for culturing over 14 days. For $10 \mathrm{~min}$, the cells were fixed to $95 \%$ ethanol. Giemsa (Sigma) staining was performed for $15 \mathrm{~min}$. The number of colonies with $>50$ cells and a diameter 
A

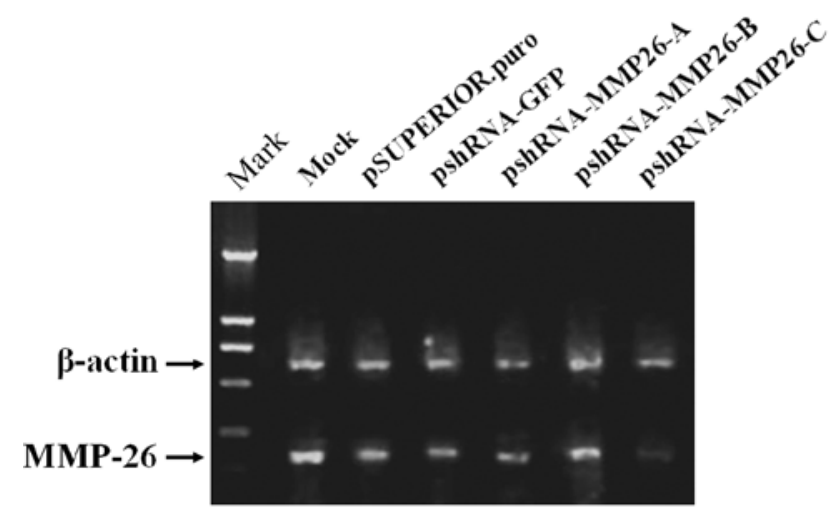

B

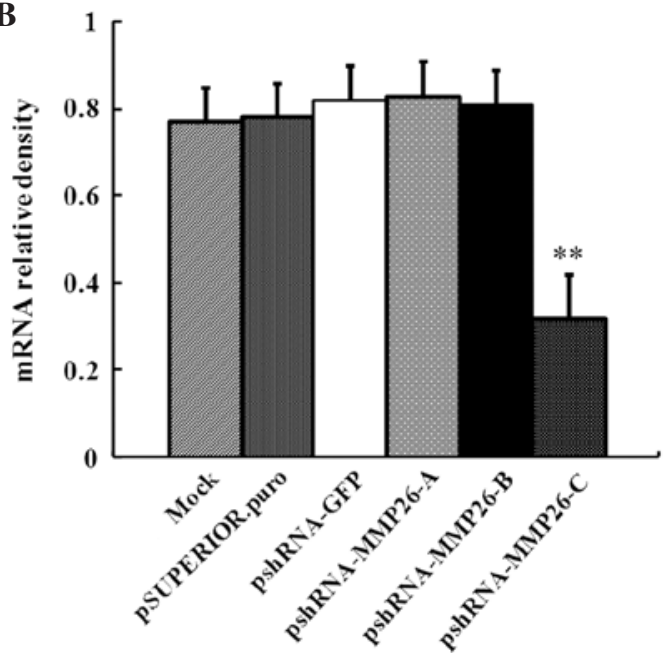

Figure 1. Semi-quantitative RT-PCR analysis of mRNA expression for MMP-26 in lung carcinoma A549 cells after transfection for $72 \mathrm{~h}$. (A) Products of a typical RT-PCR were subjected to $1.5 \%$ agarose gel electrophoresis. Lane 7 shows RNA extracts derived from parental A549 cells, A549 cells transfected with pSUPERIOR.puro, pshRNA-GFP and pshRNA-MMP26-A, -B and -C, respectively. (B) Statistical analysis by ANOVA for the semi-quantitative RT-PCR. The value of MMP-26 was normalized to that of $\beta$-actin, and the relative amount is shown as the mean $\pm \mathrm{SD} .{ }^{* *}$ Comparison with the value of parental A549 cells and A549 cells transfected with pSUPERIOR.puro. $\mathrm{P}<0.01$.

of $>0.5 \mathrm{~mm}$ were counted and averaged under an inverted microscope. The inhibition rate of colony formation was then calculated. The colony-forming inhibition rate $(\%)$ was determined using the formula: (1-the number of colonies in the test group/the number of colonies in the control group) $\mathrm{x} 100 \%$. Each experiment included 6 samples and the average value was used.

Flow cytometry. The transfected cells were cultured for $48 \mathrm{~h}$ and single cell suspensions were prepared. Cold ethanol (70\%) was used overnight for fixation. The cells were washed with phosphate-buffered saline (PBS) twice. Then, $500 \mu 1$ of PI solution (Sigma) was added, and the cells were incubated for at least $30 \mathrm{~min}$ at $4^{\circ} \mathrm{C}$ in the dark. Flow cytometry was used for detection of the cell cycle and apoptosis. Each experiment included 6 samples and the average value was used.

In vitro cell adhesion assay. Cells in the logarithmic phase were collected and single cell suspensions were prepared. The cells were seeded in 96-well plates with $1.0 \times 10^{5}$ cells in each well. After culturing for 30, 60, 90 and $120 \mathrm{~min}$, PBS was used for washing non-adherent cells. MTT was used, and the A490 cells were determined with a microplate reader. Culture medium without cells was used as a control. The cell adhesion rate was determined using the formula: (A-value in experimental group/A value in control group) $\mathrm{x} 100 \%$. Each experiment included 6 samples and the average value was used.

In vitro migration assay. Single cell suspensions were prepared until the wells were covered with cells. Scratch damage was inflicted on the cell surface with one scratch in each hole. The width was controlled to $0.7 \mathrm{~mm}$. PBS was used to wash the scratch 3 times. Wound healing was observed and visualized under an inverted microscope 0,12 and $24 \mathrm{~h}$ later. Photoshop was used to measure the size of the cell-free region at $0 \mathrm{~h}$ (A-value) and the cell-free region at different time points ( $\mathrm{B}$ value). The cell migration rate was determined using the formula: (A-B)/A x 100\%. Each experiment included 6 samples and the average value was used.

In vitro cell invasion assay. A total amount of $200 \mu \mathrm{l}$ cell suspension was added to the upper layer of a Boyden chamber (BD Biosciences, San Jose, CA, USA). Pre-cooling gel $(40 \mu \mathrm{l})$ was then added on the cellulose acetate microporous membrane. Following that, $400 \mu 1$ of supernatant of NIH3T3 cells cultured in serum-free culture medium was used as a chemokine and added to the lower layer of the Boyden chamber. Following normal culture for $48 \mathrm{~h}$, the membrane was removed and fixed with iced formaldehyde for $1 \mathrm{~min}$. Giemsa staining was then performed. A total of 5 fields were randomly selected. Cells that had migrated to the back of the polyester membrane were counted under a light microscope. The percentage of cells passing through the membrane indicates the cell invasion ability. The transmembrane cell percentage was determined using the formula: the number of cells passing through the microporous membrane covered by matrigel/the number of cells passing through the microporous membrane not covered by matrigel x $100 \%$. Each experiment included 6 samples and the average value was used.

Establishment of three-dimensional (3D) culture model in vitro. Rat tail collagen was extracted according to Schor's method (8). The collagen concentration was determined by UV spectrophotometry. Refined collagen $(0.3 \mathrm{mg} / \mathrm{ml})$, 5X H-DMEM (without serum), and the 3D buffer were mixed on ice at a ratio of $7: 2: 1$. The concentration of cells in different groups was adjusted to $5.0 \times 10^{4} / \mathrm{ml}$. Then, $200 \mu \mathrm{l}$ of cell suspension and $400 \mu \mathrm{l}$ of collagen gel were mixed. The mixture was seeded in 24-well plates. A total of 5 double holes were set in each group. The cells were cultured under normal conditions and observed and visualized every $12 \mathrm{~h}$.

Double fluorescent immunocytochemistry. After cells were attached to the cover glass, distilled water was used for $30 \mathrm{~min}$ 
A

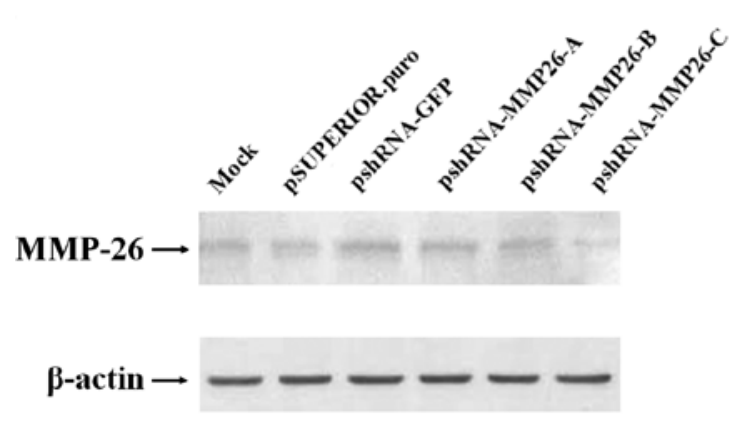

B

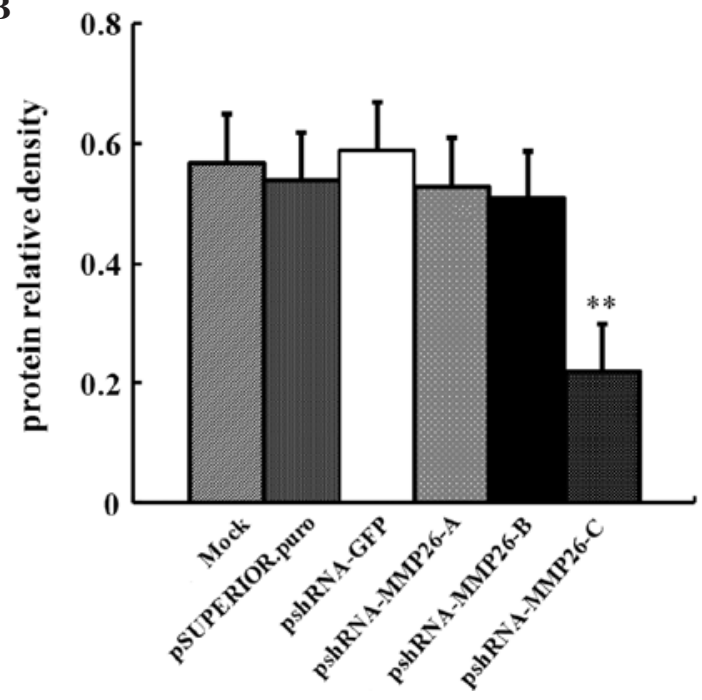

Figure 2. Western blot analysis of protein expression for MMP-26 in lung carcinoma A549 cells after transfection for $72 \mathrm{~h}$. (A) A typical result of Western blotting. Lane 6 shows protein extracts derived from parental A549 cells, A549 cells transfected with pSUPERIOR.puro, pshRNA-GFP and pshRNA-MMP26-A, $-B$ and -C, respectively. (B) Western blot analysis was performed by ANOVA. The value of MMP-26 was normalized to that of $\beta$-actin, and the relative density is shown as the mean \pm SD. ${ }^{* *}$ Comparison with the value of parental A549 cells and A549 cells transfected with pSUPERIOR.puro. P<0.01.

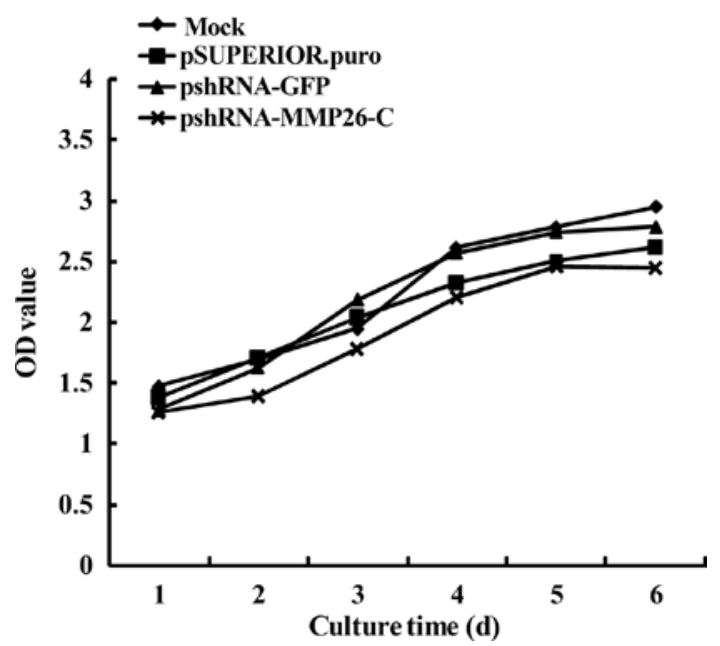

Figure 3. Effect of siRNA-MMP26 on cell proliferation of lung carcinoma A549 cells as measured by MTT. Statistical analysis by ANOVA over 6 days for the MTT assay. The data are shown as the mean \pm SD.
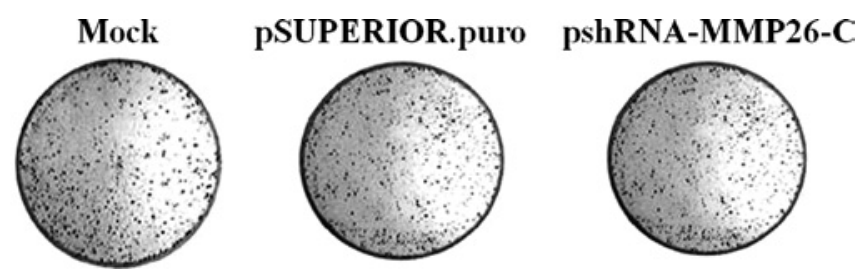

Figure 4. A typical result of the colony formation assay as determined by Giemsa staining.

of hydration. Triton X-100 (0.2\%) was incubated with the glass for 10 min and 5\% BSA was used for blocking at room temperature for 45 min. MMP-26 polyclonal antibody (1:100) or goat anti-human MMP-9 polyclonal antibody (1:400) was used for incubation at $4^{\circ} \mathrm{C}$ overnight. In the negative control, the
Table I. Effect of siRNA-MMP26 on colony formation of lung carcinoma A549 cells.

\begin{tabular}{lcc}
\hline Group & No. of colonies & $\begin{array}{c}\text { Inhibition rate of } \\
\text { colony formation (\%) }\end{array}$ \\
\hline Mock & $152 \pm 12$ & 0.0 \\
pSUPERIOR.puro & $146 \pm 10$ & 3.9 \\
pshRNA-MMP26-C & $138 \pm 8$ & 9.2 \\
\hline
\end{tabular}

first antibody was substituted with PBS. FITC- or Cy3-labeled secondary antibodies (Sigma-Aldrich) were used for incubation at room temperature for $1 \mathrm{~h}$ in the dark. The sections were then mounted with glycerol. The observation was performed under a laser scanning confocal microscope.

Statistical analysis. Data are presented as the mean \pm standard deviation (SD). SPSS11.0 software was used for data processing. Analysis of variance and the $\chi^{2}$ test were used for comparison of the means and rates, respectively. $\mathrm{P}<0.05$ or $\mathrm{P}<0.01$ were considered to be statistically significant.

\section{Results}

Effect of RNAi on MMP-26 gene and protein expression in A549 cells. The inhibitory effect was observed $36 \mathrm{~h}$ after transfection, and the effect peaked $72 \mathrm{~h}$ later. The RT-PCR results showed that the brightness of the PCR amplification band in the pshRNA-MMP26-C group exhibited a significant decrease as compared to the control, empty vector, pshRNAGFP, and pshRNA-MMP26-A and -B groups (Fig. 1A). The inhibition rate was $\sim 65 \%$ (Fig. 1B). The Western blot results showed that pshRNA-MMP26-C significantly inhibited 
Table II. Effect of siRNA-MMP26 on cell cycle and apoptosis of lung carcinoma A549 cells (\%).

\begin{tabular}{lcccc}
\hline Group & G0/G1 phase & S phase & G2/M phase & Apoptosis index \\
\hline Mock & 70.90 & 20.64 & 8.46 & 1.24 \\
pSUPERIOR.puro & 68.71 & 22.43 & 8.86 & 1.31 \\
pshRNA-MMP26-C & 67.75 & 24.27 & 7.98 & 0.88 \\
\hline
\end{tabular}
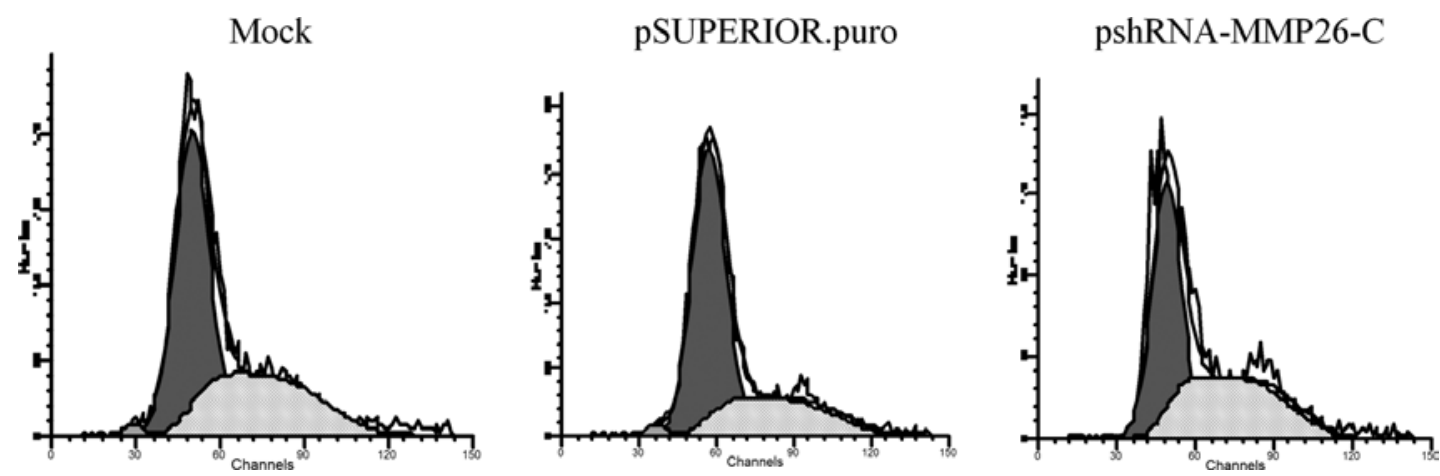

Figure 5. A typical result of cell cycle determination as detected by flow cytometry for stable transfection.

MMP-26 protein expression, which had a significant difference compared with the control group $(\mathrm{P}<0.01$, Fig. $2 \mathrm{~A})$. The inhibition rate was similar to the mRNA inhibition (Fig. 2B), indicating that the recombinant vector pshRNA-MMP26-C inhibited MMP-26 gene and protein expression on the mRNA and translational levels.

The transfection efficacy was determined by the above experiments. Single cell clones were then selected for expansion cultivation by puromycin selection. After 10 passages, RT-PCR and Western blot analysis showed that the interference effect was evident, indicating that MMP-26 siRNA had been successfully transfected into A549 cells and exhibited a stable low expression.

Effect of MMP-26 gene silencing on the biological characteristics and behavior of A549 cells. Recombinant plasmid transfected cells were collected, and the A490 cells were detected using the MTT assay for 6 consecutive days. The results showed that the absorbance gradually increased with culture time. However, the absorbance at the same time point between the two groups showed no significant differences (P>0.05, Fig. 3).

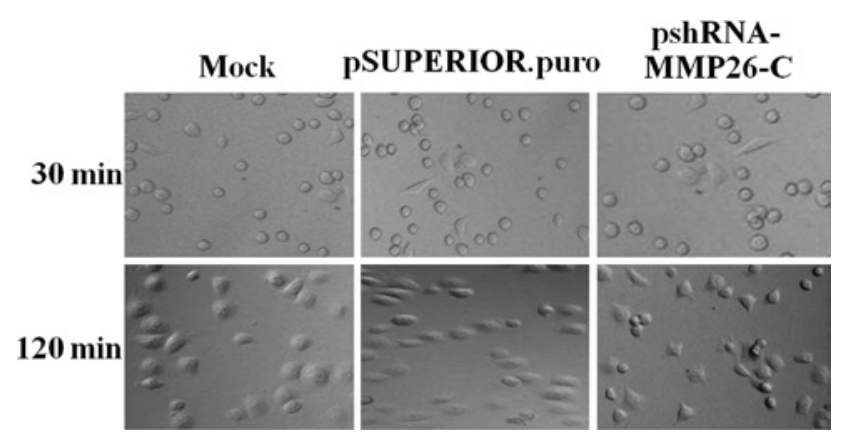

Figure 6. A typical result of the in vitro adhesion assay observed at 30 and 120 min under a light microscope at a magnification of $\mathrm{x} 200$.

The adherent cell colony forming assay results showed that the number of colonies in the control, empty vector and intervention groups were $152 \pm 12,146 \pm 10$, and $138 \pm 8$, respectively. Compared with other groups, the colony formation inhibition rate in the intervention group showed no significant difference (P>0.05, Fig. 4, Table I).

The flow cytometry results showed no significant subdiploid (apoptotic peak) in the intervention group, and the

Table III. Effect of siRNA-MMP26 on cell adhesion of lung carcinoma A549 cells $(\%, \overline{\mathrm{x}} \pm \mathrm{sec})$.

\begin{tabular}{lcccc}
\hline Group & $30 \mathrm{~min}$ & $60 \mathrm{~min}$ & $90 \min$ & $120 \mathrm{~min}$ \\
\hline Mock & $6.21 \pm 0.16$ & $18.75 \pm 2.28$ & $35.14 \pm 3.64$ & $57.31 \pm 5.51$ \\
pSUPERIOR.puro & $6.33 \pm 0.09$ & $16.53 \pm 2.61$ & $32.89 \pm 3.31$ & $54.68 \pm 6.42$ \\
pshRNA-MMP26-C & $6.11 \pm 0.23$ & $15.88 \pm 2.72$ & $26.08 \pm 2.60^{\mathrm{a}}$ & $40.28 \pm 4.15^{\mathrm{a}}$
\end{tabular}

${ }^{\text {a }}<0.05$, compared with the value of parental A549 cells and A549 cells transfected with pSUPERIOR.puro. 
Table IV. Effect of siRNA-MMP26 on cell migration of lung carcinoma A549 cells $(\%, \bar{x} \pm \mathrm{sec})$.

\begin{tabular}{lcc}
\hline Group & $12 \mathrm{~h}$ & $24 \mathrm{~h}$ \\
\hline Mock & $68.7 \pm 6.1$ & $90.2 \pm 5.7$ \\
pSUPERIOR.puro & $66.3 \pm 5.8$ & $91.3 \pm 4.2$ \\
pshRNA-MMP26-C & $64.9 \pm 7.1$ & $89.7 \pm 5.3$
\end{tabular}
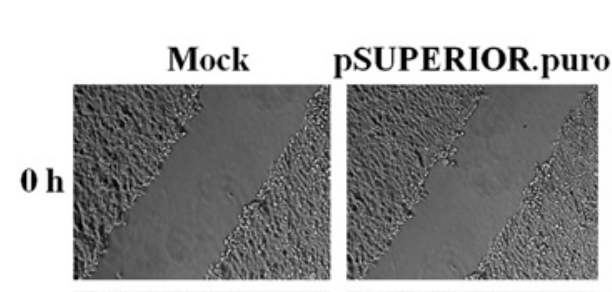

\section{pshRNA-}

$12 \mathrm{~h}$
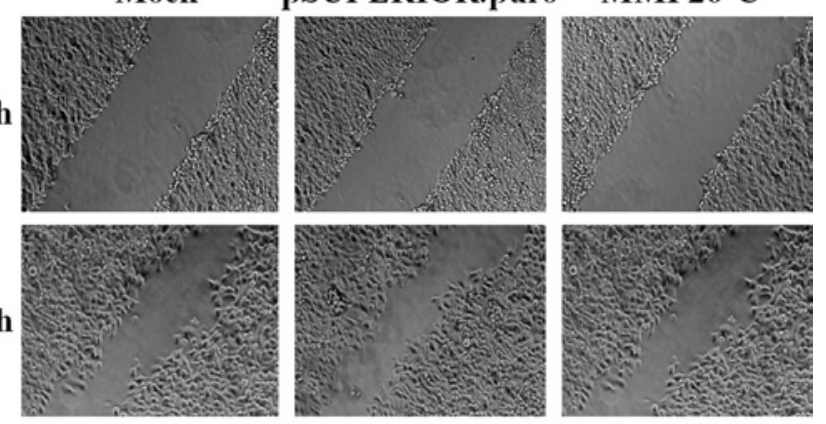

$24 \mathrm{~h}$
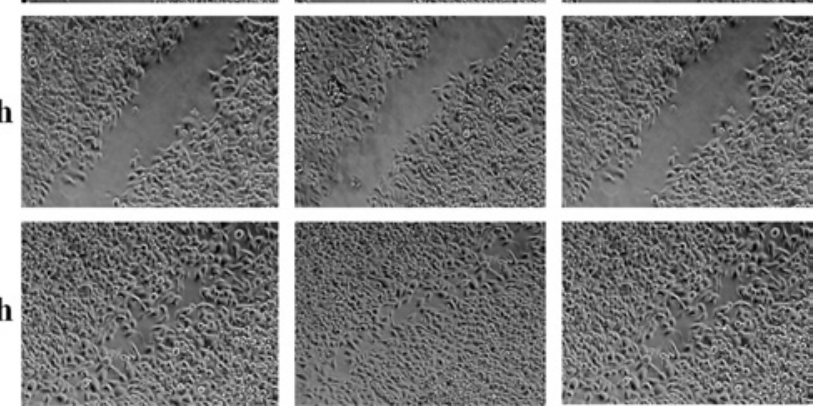

Figure 7. A typical result of the in vitro migration assay observed at 0,12 and $24 \mathrm{~h}$ under a light microscope at a magnification of $\mathrm{x} 40$.

apoptotic rate did not significantly change (Fig. 5). The cell cycle changes in the intervention group had no significant difference compared with the control group and the empty plasmid group $(\mathrm{P}>0.05$, Table II).

As shown in Fig. 6 and Table III, the cell adhesion rate on the matrigel matrix at 90 and $120 \mathrm{~min}$ in the intervention group was significantly lower than the control group $(\mathrm{P}<0.05)$. At each time point, the cell adhesion rate between the control and plasmid groups showed no significant differences $(\mathrm{P}>0.05)$, suggesting that the cell adhesion ability in the intervention group decreased.

As shown in Fig. 7 and Table IV, $12 \mathrm{~h}$ after seeding, cells in each group had a certain degree of 'healing' due to cell migration. Compared with the control group, the migration rate at the corresponding time in the intervention group decreased, but no significant difference was observed $(\mathrm{P}>0.05)$, indicating that inhibition of the MMP-26 expression had no effect on the migration of A549 cells.

Results of the cell invasion assay showed that the invasion ability in a Transwell room covered with matrigel gel (collagen IV) in the control and empty vector groups was stronger than that in the intervention group (Fig. 8A). If the invasion rate in the control group was set at $100 \%$, the invasion rate in the intervention group was $31.7 \pm 2.1 \%$. Additionally, a significant difference was observed between the two groups ( $\mathrm{P}<0.05$, Fig. 8B).

In vitro three-dimensional (3D) culture (Fig. 9) showed that in the first 3-4 h, most cells in the 3D collagen were
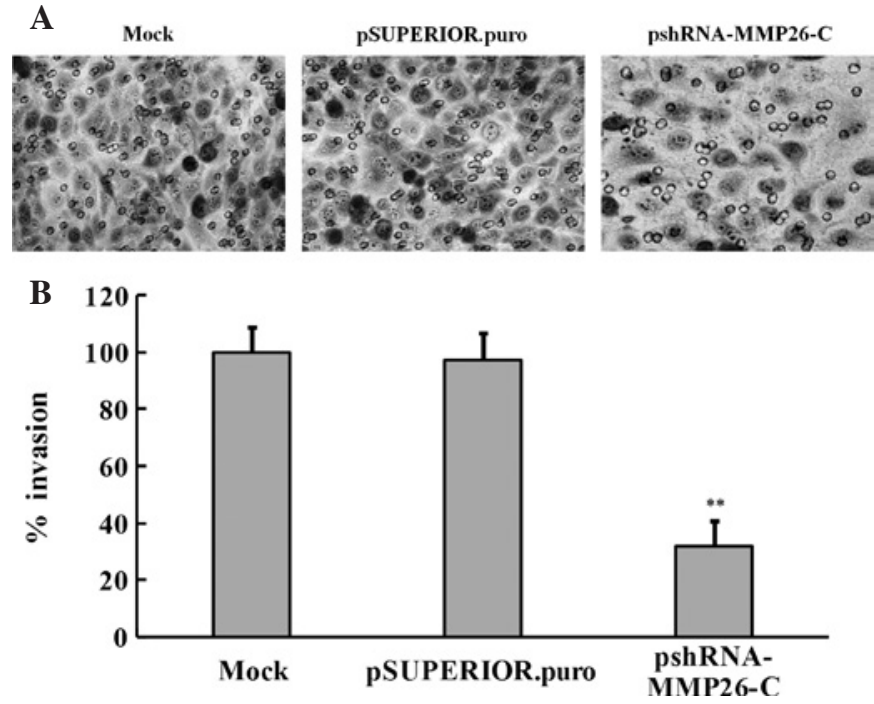

Figure 8. Effect of siRNA-MMP26 on cell invasion of lung carcinoma A549 cells. (A) A typical result of the in vitro invasion assay observed under a light microscope at a magnification of $\mathrm{x} 40$. (B) Statistical analysis by ANOVA for the invasion assay. The cell numbers of the invaded parental cells were used as $100 \%$ invasiveness. Data are shown as the mean \pm SD ${ }^{* *}$ Comparison with the value of parental A549 cells and A549 cells transfected with pSUPERIOR.puro, $\mathrm{P}<0.01$.

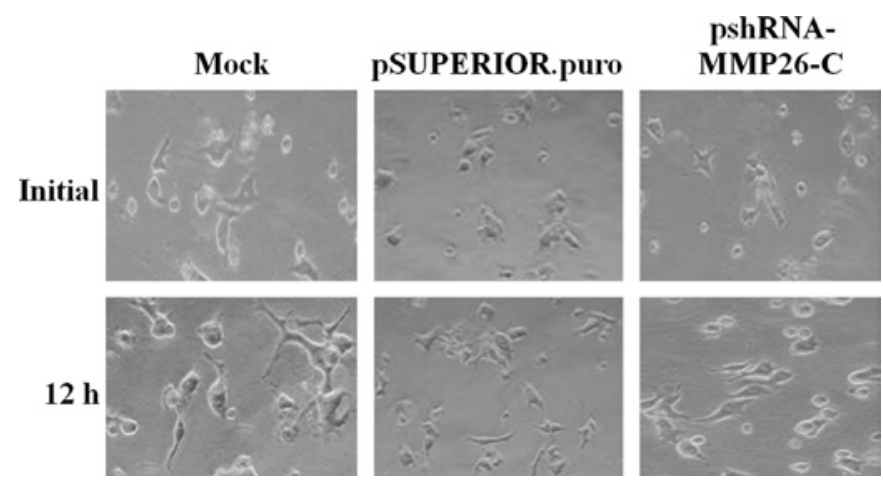

Figure 9. Morphological changes of human lung carcinoma A549 cells after stable transfection in 3D culture. Original magnification, $\mathrm{x} 200$.

round, while some cells were integrated as cell clusters with 4-10 cells in each cluster, although the configuration of single cells was clearly identified. After $12 \mathrm{~h}$, cells in the empty vector and control groups formed large and irregular colonies (certain cells formed cell clusters composed of 5-30 cells). The cells were connected loosely with irregular borders, and many pseudopods had infiltrated into the surrounding areas, although a network structure had not formed. However, in the intervention group, a network structure was formed with fewer proliferating colonies, and the border was clear and smooth. Additionally, the cells were closely connected with pseudopodia. Moreover, the proportion of round cells was increased significantly, while the level of spindle cells was significantly reduced.

To exclude the effect of siRNAs on the biological characteristics and behavior of cells, a GFP control was set and the results showed that the GFP group had no effect on the biological characteristics and behavior of the cells. 

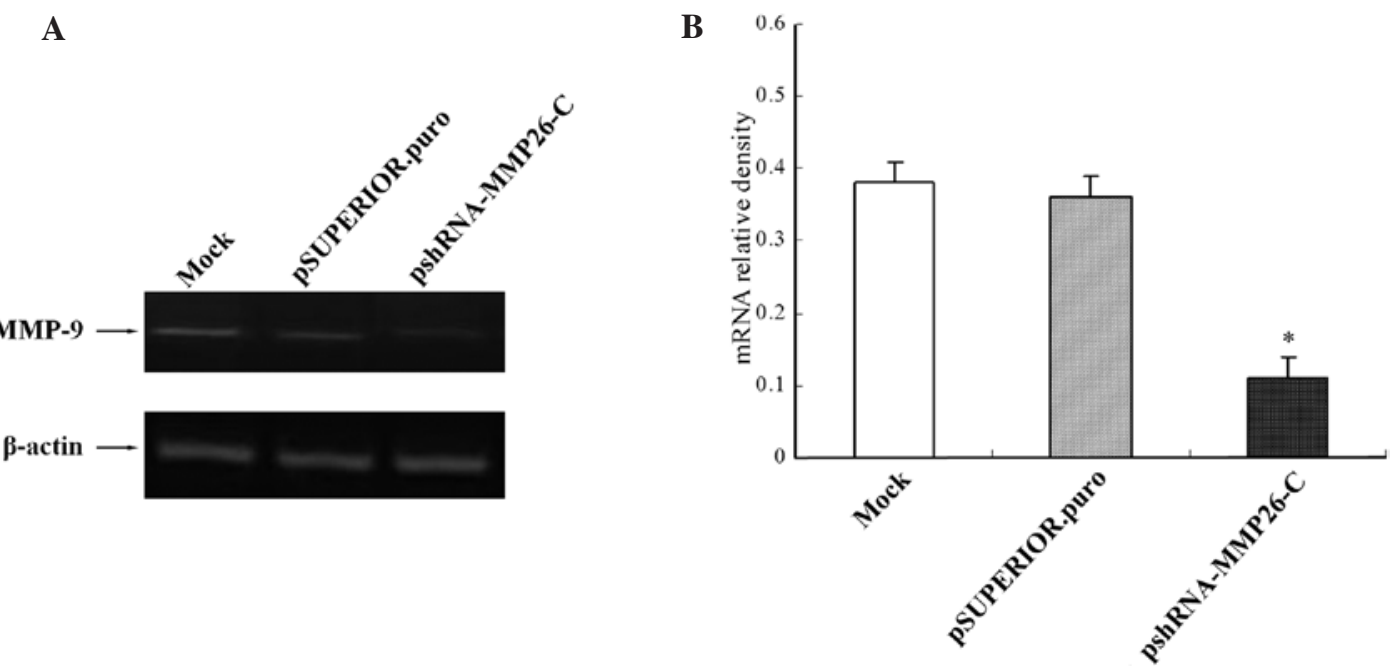

Figure 10. Semi-quantitative RT-PCR analysis of mRNA expression for MMP-9 in lung carcinoma A549 cells. (A) Products of a typical RT-PCR were subjected to $1.5 \%$ agarose gel electrophoresis. (B) Statistical analysis by ANOVA for the semi-quantitative RT-PCR. The value of MMP-9 was normalized to that of $\beta$-actin, and the relative amount is shown as the mean $\pm \mathrm{SD}$. "Comparison with the value shown by bar $2, \mathrm{P}<0.05$. Bars 1,2 and 3 are the parental A549 cells, A549 cells transfected with pSUPERIOR.puro plasmid, and A549 cells transfected with pshRNA-MMP26-C plasmid, respectively.

A

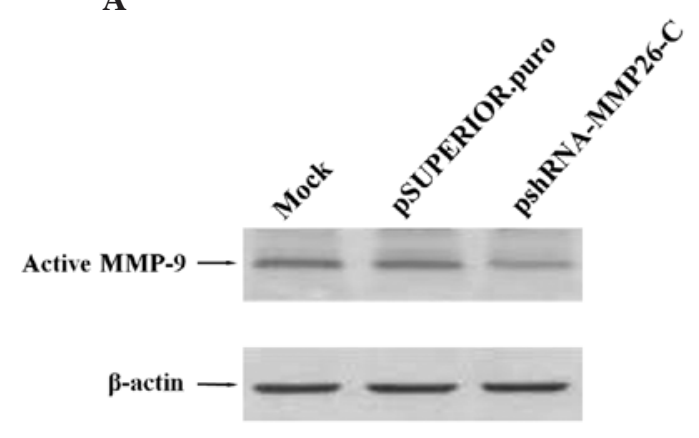

B

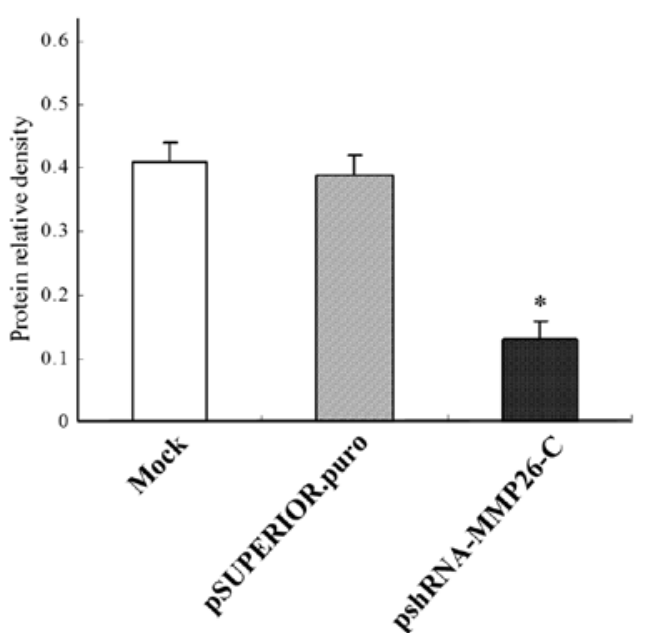

Figure 11. Western blot analysis of protein expression for MMP-9 in lung carcinoma A549 cells. (A) A typical result of Western blotting. (B) Western blot analysis was performed by ANOVA. The value of MMP-9 was normalized to that of $\beta$-actin, and the relative amount is shown as the mean \pm SD. ${ }^{*}$ Comparison with the value of bar 2, P<0.05. Bars 1, 2 and 3 represent parental A549 cells, A549 cells transfected with pSUPERIOR.puro plasmid and A549 cells transfected with pshRNA-MMP26-C plasmid, respectively.
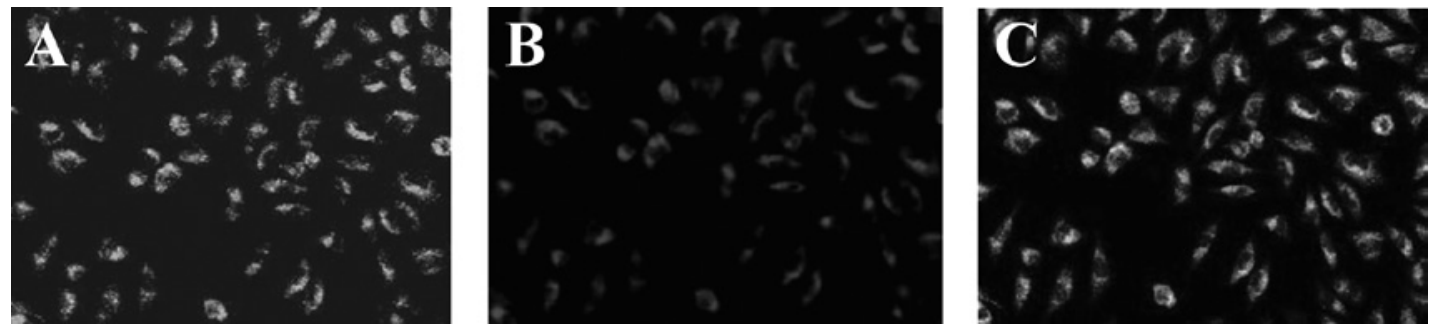

Figure 12. Double immunofluorescent staining to show the localization of MMP-26 and MMP-9 in lung carcinoma A549 cells. (A) Immunoreactivity of MMP-26 is shown. (B) The immunostaining of MMP-9 is shown. (C) Overlapping of (A) and (B). Original magnification, x 200.

Effect of MMP-26 gene silencing on the expression of MMP-9 by A549 cells. The semi-quantitative RT-PCR results (Fig. 10) showed that MMP-9 mRNA levels in the intervention group decreased compared with parental cells and empty vector transfected cells, with a reduction of approximately $60 \%$. 
The Western blot results (Fig. 11) showed that the A549 cell line expressed low levels of MMP-9 and that extremely low levels of MMP-9 protein were expressed in the intervention group.

Double immunofluorescence staining showed (Fig. 12) that MMP-26 and MMP-9 were expressed by parental and empty vector-transfected cells, with the positive signals located mainly in the cytoplasm. Confocal laser scanning microscopy image analysis revealed that the majority of MMP-26 and MMP-9 signals were overlapped, indicating colocalization of the two proteins in A549 cells.

\section{Discussion}

As a new member of the MMP family, MMP-26 has captured the attention of various investigators due to its unique molecular structure and high expression in epithelial malignant tumors. The expression and clinical significance of MMP-26 protein in NSCLC patients has been reported, and it has been suggested that MMP-26 is a tumor marker for monitoring progression and predicting prognosis of NSCLC (9).

In this study, we successfully constructed a siRNA eukaryotic expression vector targeting the MMP-26 gene and selected A549 cells as the target cell line for gene silencing transfection. MMP-26 mRNA and protein expression were determined by RT-PCR and Western blot analysis 48 and $72 \mathrm{~h}$ following transfection. The results at $48 \mathrm{~h}$ were less favorable than those at $72 \mathrm{~h}$, indicating that assessment of the RNAi effects $72 \mathrm{~h}$ after transfection was appropriate. Although the MMP-26 interference sequence existed in the transfected cells, RT-PCR and the Western blot analysis revealed marked inhibitory effects only in the pshRNA-MMP26-C group. Subsequently, A549 cells were transfected with MMP-26 and a stable monoclonal cell line was obtained after screening with $1 \mu \mathrm{g} / \mathrm{ml}$ puromycin.

Additionally, the MTT method and the colony forming assay were used for the detection of changes in cell proliferation in vitro. The results revealed that inhibition of the MMP-26 expression had no significant effect on the proliferation of A549 cells. Flow cytometry showed that although MMP-26 was regulated by the Wnt signaling pathway, members of the signaling pathway were closely related to tumor cell cycle regulation and apoptosis. Thus, the cell cycle was not affected after silencing the expression of MMP-26, and cell apoptosis was not induced.

To effectively observe the invasion and metastasis of A549 cells in vivo and their relationship, we studied four aspects as follows. The plate adhesion assay revealed that RNAi had a marked inhibitory effect on the adhesion of A549 cells in vitro. It is speculated that MMP-26 may damage or weaken the adhesion of epithelial tumor cells and increase the adhesion of the cells and ECM, thereby promoting the epithelial-mesenchymal transition (EMT) and stimulating tumor cell migration and invasion. The in vitro scratch 'healing' model showed that MMP-26 gene silencing did not affect the migration of A549 cells. This conclusion differs from the expected results and may have been caused by the serum in the cell culture medium, although cell proliferation cannot be excluded. The Transwell cell culture model showed that MMP-26 gene silencing significantly reduced the invasive ability of A549 cells. MMP-26 promotes tumor cell invasion ability, which has been confirmed in human prostate cancer ARCaP (10), human choriocarcinoma JEG-3 (11) and human glioma U251 cells (12). Results of the 3D cell culture model experiments demonstrated that in the control and empty vector groups, large and irregular colonies were formed. In addition, the cells were connected loosely with irregular borders and many pseudopods had infiltrated to the surrounding areas. Cells in the intervention group formed a network structure rather than colonies, and the colonies were clear, smooth and spherical. These results strongly support the conclusion that MMP-26 is involved in the process of cancer cell invasion, as observed in the $2 \mathrm{D}$ culture system.

It is widely recognized that MMP-26 itself may not be directly involved in the regulation of cell invasion behavior. However, if Ala93-Met94 sites of pro MMP-9 are cleaved and MMP-9 is activated, the active forms of MMP-9 may be produced, thereby promoting tumor cell invasion and migration. Zhao et al (10) observed the coexpression of MMP-26 and MMP-9 in ARCaP cells. Additionally, specific antiMMP-26 and anti-MMP-9 antibodies reduced the invasion of FN or collagen IV by ARCaP cells. MMP-26 antisense cDNA is capable of reducing the expression of MMP-26 protein by ARCaP cells, reducing activated MMP-9 secretion, and inhibiting ARCaP cell infiltration. Yamamoto et al (13) transfected MMP-26 cDNA into the ESCC cell line TE-1 and found enhanced activity of MMP-9 and in vitro invasiveness.

Thus, MMP-9-mediated ECM degradation plays a crucial role in cancer invasion and metastasis. Its relationship with lung cancer invasion and metastasis has been confirmed in several studies. Rao et al (14) transfected antisense uPAR and antisense MMP-9 adenovirus vector (Ad-uPAR-MMP-9) genes into H1299 cells, and observed that MMP-9 mRNA and protein levels were significantly reduced, while the in vitro invasion ability was significantly inhibited. Thus, MMP-9 may become a molecular target for the invasion and metastasis of NSCLC. Findings of other studies showed that the decrease in tumor cell MMP-26 mRNA and protein expression through RNAi technology was accompanied by a reduction of MMP-9 mRNA and protein. Double fluorescent immunocytochemistry revealed that MMP-26 and MMP-9 were colocalized in the cells. Therefore, we considered that an inhibited expression of MMP-26 in A549 cells may change the cellular micro-environment, thereby decreasing the expression of MMP-9 and inhibiting tumor cell invasion and metastasis. However, more experimental investigation is required to confirm this hypothesis.

\section{References}

1. Ferlay J, Bray F and Pisani P (eds): GLOBOCAN 2002: Cancer Incidence, Mortality and Prevalence Worldwide. IARC CancerBase No. 5. Version 2.0. IARC Press, Lyon, 2004.

2. Martin J, Ginsberg RJ, Venkatraman ES, et al: Long-term results of combined-modality therapy in resectable non-small-cell lung cancer. J Clin Oncol 20: 1989-1995, 2002.

3. Marchenko GN, Ratnikov BI, Rozanov DV, Godzik A, Deryugina EI and Strongin AY: Characterization of matrix metalloproteinase-26, a novel metalloproteinase widely expressed in cancer cells of epithelial origin. Biochem J 356: 705-718, 2001.

4. Marchenko ND, Marchenko GN and Strongin AY: Unconventional activation mechanisms of MMP-26, a human matrix metalloproteinase with a unique PHCGXXD cysteineswitch motif. J Biol Chem 277: 18967-18972, 2002. 
5. Park HI, Turk BE, Gerkema FE, Cantley LC and Sang QX Peptide substrate specificities and protein cleavage sites of human endometase/matrilysin-2/matrix metalloproteinase-26. J Biol Chem 277: 35168-35175, 2002.

6. Zhao YG, Xiao AZ, Ni J, Man YG and Sang QX: Expression of matrix metalloproteinase-26 in multiple human cancer tissues and smooth muscle cells. Ai Zheng 28: 1168-1175, 2009.

7. Elbashir SM, Lendeckel W and Tuschl T: RNA interference is mediated by 21- and 22-nucleotide RNAs. Genes Dev 15 $188-200,2001$.

8. Schor SL, Schor AM, Winn B and Rushton G: The use of threedimensional collagen gels for the study of tumour cell invasion in vitro: experimental parameters influencing cell migration into the gel matrix. Int J Cancer 29: 57-62, 1982.

9. Li L, Mei TH, Zhou XD and Zhang XG: Expression and clinical significance of matrix metalloproteinase (MMP)-26 protein in non-small cell lung cancer. Ai Zheng 28: 60-63, 2009.

10. Zhao YG, Xiao AZ, Newcomer RG, et al: Activation of progelatinase B by endometase/matrilysin-2 promotes invasion of human prostate cancer cells. J Biol Chem 278: 15056-15064 2003.
11. $\mathrm{Wu} \mathrm{XQ}, \mathrm{Li} \mathrm{YX}$ and $\mathrm{Fu} \mathrm{YY}$ : Effect of matrix metalloproteinase-26 on cell invasiveness in human choriocarcinomal cell line JEG-3. Prog Biochem Biophys (in Chinese) 33: 524-530, 2006.

12. Deng Y, Li W, Li Y, Yang H, Xu H, Liang S and Zhang L: Expression of Matrix Metalloproteinase-26 promotes human glioma U251 cell invasion in vitro and in vivo. Oncol Rep 23: 69-78, 2010.

13. Yamamoto H, Vinitketkumnuen A, Adachi Y, et al: Association of matrilysin-2 (MMP-26) expression with tumor progression and activation of MMP-9 in esophageal squamous cell carcinoma. Carcinogenesis 25: 2353-2360, 2004.

14. Rao JS, Gondi C, Chetty C, Chittivelu S, Joseph PA and Lakka SS: Inhibition of invasion, angiogenesis, tumor growth, and metastasis by adenovirus-mediated transfer of antisense uPAR and MMP-9 in non-small cell lung cancer cells. Mol Cancer Ther 4: 1399-1408, 2005. 